\title{
A game of pool, a game of tag: AMPA trafficking in the post-synaptic density
}

\author{
Fidel Santamaria ${ }^{1 *}$, George J Augustine ${ }^{2}$, Sridhar Raghavachari ${ }^{2}$ \\ From Nineteenth Annual Computational Neuroscience Meeting: CNS*2010 \\ San Antonio, TX, USA. 24-30 July 2010
}

One mechanism of information storage in neurons is the plasticity in the strength of synaptic contacts. The strength of an excitatory synapse is partially due to the concentration of a particular type of ionotropic glutamate receptor (AMPAR) in the post-synaptic density (PSD). AMPAR concentration in the PSD has to be plastic, to allow the storage of new memories; but it also has to be stable to preserve important information. Although much is known about the molecular identity of synapses, the biophysical mechanisms by which AMPAR can enter, leave and remain in the synapse are unclear.

We implemented Monte Carlo simulations to determine the influence of PSD structure and activity in maintaining homeostatic concentrations of AMPARs in the synapse. The model consisted of tracking the movement of individual AMPAR in and around a PSD. The membrane was simulated as a square mesh in which each position could be present a diffusing AMPAR, and anchored PSD molecule, or be empty. PSD molecules could be active, in which case, they could bind with a certain binding energy to AMPAR. If PSD molecules were inactive they acted as obstacles $[1,2]$.

We found that, the high concentration and excluded volume caused by PSD molecules result in molecular crowding. Diffusion of AMPAR in the PSD under such conditions is anomalous. Anomalous diffusion due to molecular crowding is a physical process fundamentally different from the steady-state excluded volume analyses such as tortuosity [3]. Anomalous diffusion of AMPAR results in retention of these receptors inside the PSD for periods ranging from seconds to several hours in the absence of any binding of receptors to PSD molecules. Our simulations were capable of reproducing several recent experimental results $[4,5]$. Trapping of receptors in the PSD by crowding

\footnotetext{
* Correspondence: fidel.santamaria@utsa.edu

${ }^{1}$ Department and Neurosciences Institute, University of Texas at San Antonio, San Antonio, TX 78249 USA
}

effects was very sensitive to the concentration of PSD molecules, showing a switch-like behavior for retention of receptors. Non-covalent binding of AMPAR to anchored PSD molecules allowed the synapse to become well-mixed, resulting in normal diffusion of AMPAR. Binding also allowed the exchange of receptors in and out of the PSD.

We propose that molecular crowding is an important mechanism to maintain homeostatic synaptic concentrations of AMPARs in the PSD without the need of energetically expensive biochemical reactions. Binding of AMPAR with PSD molecules could collaborate with crowding to maintain synaptic homeostasis but could also allows synaptic plasticity by increasing the exchange of these receptors with the surrounding extra-synaptic membrane.

\section{Acknowledgements}

NSF0932339 to FS, NSF064200 to SR and HFSP to GA.

\section{Author details}

'Department and Neurosciences Institute, University of Texas at San Antonio, San Antonio, TX 78249 USA. ${ }^{2}$ Department of Neurobiology, Duke University Medical Center, P.O. Box 3209, Durham, NC, 27710, USA.

Published: 20 July 2010

\section{References}

1. Saxton MJ: Anomalous diffusion due to binding: a Monte Carlo study. Biophys J 1996, 70(3):1250-1262.

2. Saxton MJ: Anomalous diffusion due to obstacles: a Monte Carlo study. Biophys J 1994, 66(2 Pt 1):394-401.

3. Lacks DJ: Tortuosity and anomalous diffusion in the neuromuscular junction. Phys Rev E Stat Nonlin Soft Matter Phys 2008, 77(4 Pt 1):041912.

4. Petrini EM, Lu J, Cognet L, Lounis B, Ehlers MD, Choquet D: Endocytic Trafficking and Recycling Maintain a Pool of Mobile Surface AMPA Receptors Required for Synaptic Potentiation. Neuron 2009, 63(1):92-105.

5. Ehlers MD, Heine M, Groc L, Lee MC, Choquet D: Diffusional trapping of GluR1 AMPA receptors by input-specific synaptic activity. Neuron 2007, 54(3):447-460.

doi:10.1186/1471-2202-11-S1-F2

Cite this article as: Santamaria et al:: A game of pool, a game of tag: AMPA trafficking in the post-synaptic density. BMC Neuroscience 2010 11(Suppl 1):F2. 